\title{
DESAFIOS DO ENFERMEIRO NA CONSULTA DE PUERICULTURA PARA O ESTÍMULO DO CUIDADO E AUTOCUIDADO
}

\section{ARTIGO DE REVISÃo}

TRINDADE, Cristiane Ribeiro ${ }^{1}$

SANTOS, Gustavo Gonçalves dos ${ }^{2}$

MENDONÇA, Jéssica Akamine de ${ }^{3}$

RANGEL, Sabina Dias 4

TRINDADE, Cristiane Ribeiro. Et al. Desafios do enfermeiro na consulta de puericultura para o estímulo do cuidado e autocuidado. Revista Científica Multidisciplinar Núcleo do Conhecimento. Ano 04, Ed. 07, Vol. 01, pp. 163-173. Julho de 2019. ISSN: 2448-0959

${ }^{1}$ Enfermeira. Graduada em Enfermagem pela Escola de Ciências da Saúde da Universidade Anhembi Morumbi.

${ }^{2}$ Enfermeiro e Docente. Graduado em Enfermagem pela Escola de Ciências da Saúde da Universidade Anhembi Morumbi. Pós-graduando em Enfermagem Obstétrica e Ginecológica pelo Instituto Israelita de Ensino e Pesquisa Albert Einstein. Pósgraduando Enfermagem em Saúde Pública pela Escola Paulista de Enfermagem da Universidade Federal de São Paulo.

${ }^{3}$ Enfermeira. Graduada em Enfermagem pela Escola de Ciências da Saúde da Universidade Anhembi Morumbi.

${ }^{4}$ Enfermeira. Graduada em Enfermagem pela Escola de Ciências da Saúde da Universidade Anhembi Morumbi. Residente em Emergências e Intensivismo em Neonatologia e Pediatria pela Universidade de Santo Amaro. 


\section{RESUMO}

Objetivo: Identificar as atividades e desafios que o Enfermeiro enfrenta para realizar o estímulo do cuidado e autocuidado durante a consulta de puericultura. Método: Tratase de uma revisão de literatura, estudo exploratório e descritivo. Realizada a busca de artigos científicos, foram utilizados os seguintes descritores: Cuidado da criança. Criança. Adolescente. Puericultura, a partir das bases de dados da Literatura LatinoAmericana e do Caribe em Ciências da Saúde (LILACS), Scientific Eletronic Library Online (SciELO) e Base de dados de Enfermagem (BDENF) com critério de inclusão artigos publicados no idioma Português, publicados no período de dez anos e que abordem a temática do estudo. A revisão de literatura tem a finalidade de colocar o pesquisador em contato com o que já foi publicado em relação ao tema proposto, proporcionado ao autor avaliar a temática, tendo um novo enfoque sob a Enfermagem na Saúde da criança. Resultados: A família e a criança devem ser orientadas e possui participação ativa durante as consultas de puericultura, podendo haver maior satisfação; a falta de orientação e comunicação inadequada fez com que pais e responsáveis não levassem mais seus filhos para a consulta; a insatisfação dos usuários quanto ao atendimento é quando o profissional não estimula e também não favorece o processo do cuidar e que os pais e/ou responsáveis não identificam que o Enfermeiro e a equipe multiprofissional podem realizar a consulta de puericultura, pois o protagonista principal desta história é o Médico Pediatra. Conclusões: Para que haja o estímulo do cuidado e autocuidado na consulta de puericultura é preciso reorganizar os processos das práticas assistenciais, vislumbrar a relação que temos do modelo assistencial e entendermos com clareza e fundamento as evidências no processo de saúde-doença da criança e adolescente.

Palavras-chaves: Cuidado da criança, criança, adolescente, puericultura.

\section{INTRODUÇÃO}

A gestação é um período de transformações para a mulher e para a família. O período gestacional é composto por intensas vivências e sentimentos considerados contraditórios, sendo eles, momentos de dúvidas, de ansiedade e outros diversos. Ao 
longo dos nove meses da gestação o corpo da mulher passa por mudanças lentamente, conforme o desenvolvimento do feto. ${ }^{1,2,3,4,5}$ Durante e após o parto a equipe de Enfermagem atende a mãe e o recém-nascido para um acompanhamento de rotina de todo estado físico e emocional da mãe e crescimento e desenvolvimento deste recém-nascido. ${ }^{6}$

No Brasil, segundo o Ministério de Saúde o acompanhamento do crescimento e desenvolvimento da criança, faz parte de uma avaliação integral para a Saúde da criança de zero a dezenove anos, parte desta avaliação é denominada consulta de puericultura que envolve a avaliação fisiológica e patológica da criança e do adolescente. $7,8,9$

O acompanhamento deve ser realizado de forma regular de modo que se possam avaliar as alterações precoces fisiológicas e psicológicas da criança. No Brasil, com base nas últimas pesquisas, o acompanhamento nos serviços de Saúde Pública da Atenção Primária tem sido ofertado por Enfermeiros. O acompanhamento deve ser realizado pela Atenção Primária do Sistema Único de Saúde e também deve conter todo o apoio dos familiares, comunidade e profissionais da saúde. 7,8,9

É papel de um Enfermeiro desenvolver conhecimentos necessários para a realização de uma avaliação de puericultura, tomada de decisões e orientações para os pais e/ou responsáveis pela criança. Neste contexto, o Enfermeiro deve realizar um atendimento com cuidado integrado e humanizado considerando a criança como um todo, observando o contexto socioeconômico, cultural e familiar no qual a criança está inserida. $7,8,9$

A puericultura surgiu em meados do século XVIII na França, definida como regras básicas para a vida infantil ações que tratem a respeito da alimentação e toda fisiologia da criança Esse aspecto e visão se modificaram com o decorrer do tempo e a puericultura passou a ser tratada como controle da criança e todos os aspectos que ela possa vir a desenvolver, acompanhando o crescimento e desenvolvimento e prevenção de patologias. ${ }^{3,4,7,8,9}$ 
Diante do que foi exposto, surge a seguinte indagação: Quais são os desafios do Enfermeiro na consulta de puericultura para o estímulo do cuidado e autocuidado? Tem-se como hipótese que para que haja um serviço efetivo de qualidade o profissional Enfermeiro deve possuir especialização em Saúde da Família e/ou Saúda da criança e do adolescente, possua educação continuada juntamente com sua equipe multiprofissional, realize a promoção e recuperação da saúde das crianças, pois segundo Houassis a criança deve ser acompanhada desde a gestação até a puberdade. E para que este serviço seja ofertado à população, cabe ao enfermeiro juntamente com a secretaria de saúde e higiene da cidade realizar educação em saúde com a população oferecendo então em unidades básicas de saúde consultas de puericultura para crianças e adolescentes dentro da atenção primária do Sistema único de saúde.

\section{PUERICULTURA OCULTA PARA A FAMÍLIA E COMUNIDADE}

A concepção que temos atualmente na Atenção Primaria à Saúde é o trabalho em equipe e direcionamento de ações para comunidade. A Atenção Primária neste contexto, está juntamente ligada a Estratégia Saúde da Família que reorganiza serviços e reorienta as práticas profissionais assistências de modo com que a Estratégia Saúde da Família trabalhe com ações multidisciplinares na promoção e prevenção de patologias. Há a necessidade a modificação do modelo tradicional para o modelo assistencial como foco na família de forma contínua e integral. ${ }^{1,2}$

Segundo Dixis Figueroa e pesquisas realizadas recentemente há um carência na Saúde Pública, destacando as fragilidades relacionadas à inter-relação dos profissionais, aperfeiçoamento inadequado da equipe, havendo então a necessidade de melhorias na formação e qualificação destes profissionais que atuam na Atenção primária. Estudos apontam que o estímulo do cuidado e do autocuidado não é gerado, devido à estrutura da unidade básica de saúde e falta de aperfeiçoamento dos profissionais. ${ }^{2}$

A consulta de puericultura favorece o seguimento da criança após o nascimento até os dezenove anos de idade visam por tanto à orientação antecipada de pais ou 
responsáveis, podendo realizar diagnostico precoce a prevenção de agravos à Saúde da criança e do adolescente. Para que a consulta seja efetiva o profissional Enfermeiro deve conhecer a realidade da família e da criança para atender então as suas reais necessidades. Os profissionais responsáveis pela consulta de puericultura não podem esquecer-se da importância de ações educativas e aperfeiçoamento de sua equipe, pois isto interfere no processo saúde-doença da Saúde da criança e do adolescente. 1,2

Estudos realizados em diversos estados apontam que para que o estímulo do cuidado e do autocuidado seja ofertado durante as consultas de puericultura é necessário que haja uma mudança de melhoria dentro na unidade básica e que toda a equipe seja reorientada e aperfeiçoada para desenvolver serviços de ações educativas e realizar a consulta de forma efetiva com qualidade promovendo então a saúde e a prevenção de patologias que possam vir acometer crianças e adolescentes. ${ }^{1,2}$

\section{OBJETIVOS}

Tem-se como objetivo geral descrever as ações do Enfermeiro na consulta de puericultura e como objetivo específico identificar as atividades e desafios que o Enfermeiro enfrenta para realizar o estímulo do cuidado e autocuidado durante a consulta de puericultura.

\section{MÉTODO}

\section{DESENHO, LOCAL DO ESTUDO E PERÍODO}

Trata-se de uma revisão de literatura, estudo exploratório e descritivo. Adotou-se a revisão de literatura por ser um método de pesquisa utilizado desde 1980 no âmbito de pesquisa de práticas baseadas em evidências e explanar todo conhecimento em relação à área da Enfermagem na Saúde da criança e do adolescente. 


\section{AMOSTRAS; CRITÉRIOS DE INCLUSÃO E EXCLUSÃO}

Fase 1: Foi realizada a buscar pelos DeCS (Descritores em Ciências da Saúde) na BVS (Biblioteca Virtual em Saúde). Fase 2: Foi elaborada a pergunta norteadora: Quais são os desafios do Enfermeiro na consulta de puericultura para o estímulo do cuidado e autocuidado? Fase 3: Para responder a pergunta norteadora e elaborar o manuscrito foi realizada a busca de artigos científicos, utilizando os seguintes descritores: Cuidado da criança. Criança. Adolescente. Puericultura, a partir das bases de dados da Literatura Latino-Americana e do Caribe em Ciências da Saúde (LILACS), Scientific Eletronic Library Online (SciELO) e Base de dados de Enfermagem (BDENF). Fase 4: Foram estabelecidos os critérios de inclusão e exclusão para seleção dos artigos: inclusão de publicações no idioma português, artigos que ressaltam com relevância a temática e artigos publicados nos últimos dez anos e exclusão de publicações em idiomas estrangeiros e que não abordam com relevância a temática. Fase 5: Foi realizada a busca de artigos científicos em Scientific Eletronic Library Online (SciELO) Fase 6: Foi realizada a busca de artigos científicos em Literatura Latino-Americana e do Caribe em Ciências da Saúde (LILACS) Fase 7: Foi realizada a busca de artigos científicos em Base de dados de Enfermagem (BDENF). Ainda referente à busca, foi utilizado também Livros da Saúde da criança, Cartilhas e Manuais elaborados pela Organização Mundial de Saúde e Ministério de Saúde que atendem na integra a temática do estudo. Fase 8: No final da busca de artigos científicos em todas as bases de dados: Literatura Latino-Americana e do Caribe em Ciências da Saúde (LILACS), Scientific Eletronic Library Online (SciELO), Base de dados de Enfermagem (BDENF) foram incluídos artigos científicos pois abordam com grande relevância a temática deste estudo e possui publicação no período dos últimos dez anos. Fase 9: Após a categorização e inclusão do material referencial teórico iniciou-se a analise dos resultados.

\section{ANÁLISE DOS RESULTADOS}

O percurso metodológico seguiu as seguintes etapas: formulação do problema de pesquisa, coleta dos dados, avaliação dos dados, análise e interpretação dos dados 
e apresentação dos resultados obtidos. Foi realizada a caracterização dos estudos selecionados com leitura integral dos artigos científicos que abordam com relevância a temática deste estudo e que foram publicados nos últimos cinco anos. Foram analisados, interpretados e discutidos os dados encontrados nos artigos selecionados por meio de leitura integral. Finalizou as etapas com elaboração e apresentação da presente revisão, confrontando as ideias dos autores utilizando análise teóricoreflexiva, exploratória e descritiva. A revisão de literatura tem a finalidade de colocar o pesquisador em contato com o que já foi publicado em relação ao tema proposto, proporcionado ao autor avaliar a temática, tendo um novo enfoque sob a Enfermagem na Saúde da criança.

\section{RESULTADOS E DISCUSSÃO}

Na presente revisão foram analisados os artigos e materiais do Ministério da Saúde que atenderam aos critérios de inclusão, conforme apresentado no Quadro 1. As fontes de publicação foram de variados periódicos, dentre os quais destacamos periódicos na área da Enfermagem. As publicações concentraram-se no período de 2010 a 2018. Verifica-se que a maioria das produções científicas tem origem no Brasil. Quanto à formação acadêmica dos autores, nota-se que a maioria são profissionais da área da saúde, como por exemplo, Enfermeiros, Médicos e Psicólogos. Ao analisar-se a abordagem, obtivemos estudos que utilizaram a abordagem metodológica qualitativa, estudos também com métodos quantitativos, descritivos e de reflexão acerca da temática abordada. As publicações foram produzidas em diferentes cenários, algumas das pesquisas, por exemplo, foram realizadas em serviços de saúde.

Quadro 1- Síntese de publicações incluídas na revisão integrativa, seguindo: título, autor, ano de publicação e idioma. São Paulo, 2018.

\begin{tabular}{|l|l|l|l|l|}
\hline $\begin{array}{l}\text { Estudo } \\
\text { (E) }\end{array}$ & Título & Autor & Ano & Idioma \\
\hline
\end{tabular}




\begin{tabular}{|c|c|c|c|c|}
\hline E1 & $\begin{array}{l}\text { Percepções dos familiares de } \\
\text { crianças sobre a consulta de } \\
\text { puericultura na estratégia saúde } \\
\text { da família }\end{array}$ & $\begin{array}{lr}\text { Silva } & T, \\
\text { Aparecida } & \text { M } \\
\text { Harumi I } & \end{array}$ & 2015 & Português \\
\hline E2 & $\begin{array}{l}\text { Avaliação da vigilância do } \\
\text { crescimento nas consultas de } \\
\text { puericultura na Estratégia Saúde } \\
\text { da Família em dois municípios do } \\
\text { estado da Paraíba. }\end{array}$ & $\begin{array}{lll}\text { Figueroa } & D & e \\
\text { Santos I } & & \end{array}$ & 2017 & Português \\
\hline E3 & $\begin{array}{l}\text { O pediatra, a puericultura e a } \\
\text { criança "normal" (essa } \\
\text { desaparecida) }\end{array}$ & Leone C & 1994 & Português \\
\hline E4 & $\begin{array}{l}\text { Estudo da assistência integral à } \\
\text { criança e ao adolescente através } \\
\text { da pesquisa qualitativa. }\end{array}$ & $\begin{array}{ll}\text { Rocha } & \text { SMM, } \\
\text { Lima } & \text { RAG, } \\
\text { Scochi } & \text { CGS, } \\
\text { Vendrustulo } & \text { MS }\end{array}$ & 1998 & Português \\
\hline E5 & $\begin{array}{l}\text { O cuidado de Enfermagem } \\
\text { materna }\end{array}$ & Lowdermilk LD & 2010 & Português \\
\hline E6 & $\begin{array}{l}\text { Caderneta de Saúde da Criança. } \\
\text { Passaporte da cidadania }\end{array}$ & Brasil, MS & 2015 & Português \\
\hline E7 & $\begin{array}{l}\text { Manual do acompanhamento da } \\
\text { criança. Secretaria do Estado de } \\
\text { São Paulo }\end{array}$ & Brasil, MS & 2015 & Português \\
\hline E8 & $\begin{array}{l}\text { Departamento de Atenção Básica. } \\
\text { Saúde da criança : aleitamento } \\
\text { materno e alimentação } \\
\text { complementar }\end{array}$ & Brasil, MS & 2015 & Português \\
\hline
\end{tabular}

Fonte: Dados do estudo.

A literatura baseada evidências, aponta que a família e a criança devem ser orientadas e possui participação ativa durante as consultas de puericultura, podendo haver maior 
satisfação ao tratamento e as orientações que serão passadas. Sob a ótica dos familiares sobre a consulta de puericultura, podemos apontar e observar a falta de conhecimento sobre a mesma, não sabem a finalidade e a importância da consulta de puericultura na atenção primária. $O$ termo puericultura é considerado ainda oculto, pois os profissionais da saúde e a equipe multiprofissional não realizam educação em saúde com a família e com a comunidade. 1,2

Estudos realizados no Ceará e no Rio Grande do Sul apontam a falta de orientação e comunicação inadequada fizeram com que pais e responsáveis não levassem mais seus filhos para a consulta, obtendo como resultado que a consulta de puericultura é considerada desnecessária para crianças e adolescentes, pois não estão doentes. Educação em saúde é imprescindível e deve ser promovida para a família e comunidade, tanto por meio de ações individuais como coletivas, possibilitando e facilitando mudanças significativas no cuidado prestado para a criança e adolescente. É necessário modificar e remodelar os profissionais, as equipes, a estrutura da unidade básica, aperfeiçoar os profissionais, protocolar e colocar em prática assistencial a função necessária de cada membro da equipe. ${ }^{1,2}$

Pesquisas apontam que o Enfermeiro e toda sua equipe precisam ser capacitação e reorientados para ofertar um serviço de qualidade para a família e comunidade, é necessário domínio teórico, prático e cientifico, o profissional deve possuir autonomia e coerência na realização e elaboração das práticas assistenciais. ${ }^{1,2}$

A indagação: Quais são os desafios do enfermeiro na consulta de puericultura para o estímulo do cuidado e autocuidado? Foi respondida através de pesquisas e estudos realizados recentemente que apontam que, o estimulo do cuidado e autocuidado é gerado quando a atenção primaria possuir profissional com especialização, ofertando então capacitação para a equipe, realizando educação em saúde com a população oferecendo então as famílias e a comunidade orientações com base em evidências nas práticas assistenciais de Enfermagem.

A insatisfação dos usuários quanto ao atendimento é quando o profissional não estimula e também não favorece o processo do cuidar. Existe uma barreira, uma falta 
de comunicação entre os pais e profissionais, dificultando então o desenvolvimento da consulta. Para que haja o estímulo do cuidado e do autocuidado o Enfermeiro deve possuir autoconhecimento e ter autonomia, pois estudos apontam que os pais e responsáveis não identificam que o Enfermeiro e a equipe multiprofissional podem realizar a consulta de puericultura, pois o protagonista principal desta história é o médico pediatra. Pesquisas apontam que $9 \%$ dos pais que passaram em consulta de puericultura com Enfermeiros solicitavam retorno com o médico, este comportamento reflete a restrita assistência de saúde, onde o medico é conhecido como o modelo de assistência e o Enfermeiro e a equipe multiprofissional é denotada por falta de conhecimento. ${ }^{1,2}$

Ressalta-se que de acordo com o Manual de Saúde da criança e do adolescente do Ministério de saúde a consulta de puericultura deve ser desenvolvida pelo médico, Enfermeiro e equipe multiprofissional. O Enfermeiro precisa destacar-se no processo de valorização e sistematização durante a consulta de puericultura, de forma sistematizada, humanizada, integral tendo como finalidade promover mudanças individuais e também coletivas fortalecendo o papel profissional e ampliando o foco do cuidado e autocuidado para a criança e família. $7,8,9$

O Enfermeiro necessita ser conhecedor de todas as políticas de saúde e da atenção à criança, para que consiga sua atuação na realização dos encaminhamentos que a criança precisará. $O$ enfermeiro precisa também buscar ferramentas de comunicação com a criança, sabendo interagir com ela, tornando assim a informação um processo presente e a relação com a equipe de enfermagem satisfatória. O Enfermeiro deve promover um ambiente saudável à criança. Para o enfermeiro é necessário conhecer - Estatuto da Criança e do Adolescente e agir sempre na intenção de cumprir na realização de todas as consultas. O Enfermeiro tem responsabilidade de promover a segurança no atendimento à criança em todas as suas fases. $\mathrm{E}$ cabe ao enfermeiro manter toda a sua equipe de atendimento capacitada para a prestação da assistência de enfermagem infantil. $7,8,9$

O estímulo do cuidado e do autocuidado será gerado através de ações que estabeleçam vínculos com a família e comunidade. Diversos fatores desencadeiam a 
falta do estimulo do cuidado e do autocuidado, fatores que influenciam a relação da equipe multiprofissional que não possui integrabilidade e não transmite 0 deve necessário para a família e comunidade. Os desafios do Enfermeiro para gerar o estímulo do cuidado e autocuidado na consulta de puericultura obteve resultado como: ausência da relação entre os profissionais, aperfeiçoamento inadequado dos profissionais e da equipe, falta de projetos de educação em saúde, falta de autonomia e conhecimento teórico-prático e científico e a falta de protagonismo para que as pessoas possam reconhecer cada profissional e sua respectiva função. ${ }^{1,2}$

\section{CONSIDERAÇÕES FINAIS}

Consideramos que, sobre a consulta de puericultura é revelada a falta de conhecimento a respeito de sua importância e finalidade. A consulta de puericultura é realizada por médicos e Enfermeiros, é considerado um instrumento que oferta a promoção da saúde na atenção primária. Por isto, as atividades devem ser realizadas e executadas com qualidade para a família e comunidade. Para que haja o estímulo do cuidado e autocuidado na consulta de puericultura é preciso reorganizar os processos das práticas assistenciais, vislumbrar a relação que temos do modelo assistencial e entendermos com clareza as ações baseada em evidências científicas no processo de saúde-doença.

O Enfermeiro deve assumir a responsabilidade sobre as ações de educação em saúde, capacitar sua equipe, realizar educação permanente, possuir a aperfeiçoamento de desenvolver atividades efetivas para os profissionais e comunidade. É necessário mostrar-se preparado e dominar a metodologia do respectivo assunto.É papel do Enfermeiro, realizar gestão em Enfermagem de qualidade em seu ambiente de trabalho, sabendo a real importância do trabalho em equipe, é necessário protagonizar o seu papel e a sua função, colocando em prática todo o seu conhecimento adquirido na saúde da criança e do adolescente. O Enfermeiro e profissionais da saúde devem realizar educação em saúde, palestras, eventos de orientações para a população. É direito da criança e do adolescente receber acompanhamento de seu crescimento e desenvolvimento por um enfermeiro e médico. $O$ enfermeiro deve propor intervenções em unidades básicas de saúde e 
hospitais para que haja a avaliação psicossocial, psicomotora, alimentação e higiene visando o conforto e bem estar da criança e adolescente para que haja a valorização da qualidade de vida. O profissional deve emponderar os pais ou responsáveis a estimular relação da criança e adolescente com a família e o meio social, respeitando as fases da criança e adolescente.

Para que haja o conhecimento da população referente à consulta de puericultura e valorização do Enfermeiro na pediatria devemos expor este serviço para toda a população, mostrando que todas as crianças e adolescentes têm o direito e deve ter acompanhamento a cada trinta dias por um profissional da saúde, avaliando a criança em cada fase de seu desenvolvimento. O Enfermeiro deve interagir com a criança e com a família, uma vez que todos os processos ambientais e sociais podem interferir no desenvolvimento normal da criança podem estar ligados ao convívio familiar.

\section{REFERÊNCIAS BIBLIOGRÁFICAS}

1. Malaquias TS SILVA, Munhoz MA, Harumi I. Percepções dos familiares de crianças sobre a consulta de puericultura na estratégia saúde da família. Revista Gaucha de Enfermagem RGE. Março de 2015. p. 62-68.

2. Pedraza DF, Santos I. Avaliação da vigilância do crescimento nas consultas de puericultura na Estratégia Saúde da Família em dois municípios do estado da Paraíba. Artigo original de Epidemiologia do serviço de saúde de Brasília-DF. Outubro de 2017. p. 847-855.

3. Leone C. O pediatra, a puericultura e a criança "normal" (essa desaparecida). Pediatria (São Paulo) 1994;

4. Rocha SSM, Lima RAG, Scochi CGS, Vendrúsculo DMS. Estudo da assistência integral à criança e ao adolescente através da pesquisa qualitativa. 6ํeㄹção, p. 5-15, dezembro 1998.

5. Ricco RG, Santori JR, Almeida CAN, DEL CL. Puericultura e atenção a saúde da criança e do adolescente. In: DEL CIAMPO LA, DE ALMEIDA CAN, editores. Puericultura princípios e práticas: atenção integral à saúde da criança e do adolescente. $2^{\underline{a}}$ ed. São Paulo: Atheneu; 2008. p. 1-6. 
6. Lowdermilk, LD. O cuidado de Enfermagem materna. 5 ${ }^{\mathrm{a}}$ edição. Porto Alegre, 2010. p.1-6.

7. BRASIL. Ministério da Saúde. Caderneta de Saúde da Criança. Passaporte da cidadania. 10ª edição. 2015. Brasília-DF. p. 1-90.

8. BRASIL. Ministério da Saúde. Manual do acompanhamento da criança. Secretaria do Estado de São Paulo. Agosto de 2015. p. 1-23.

9. Brasil. Ministério da Saúde. Secretaria de Atenção à Saúde. Departamento de Atenção Básica. Saúde da criança: aleitamento materno e alimentação complementar. Ministério da Saúde, Secretaria de Atenção à Saúde, Departamento de Atenção Básica. - 2. ed. - Brasília : Ministério da Saúde, 2015.

Enviado: Junho, 2019.

Aprovado: Julho, 2019. 